\title{
MOLECULAR MODELLING OF THE DEFORMATION MECHANISMS ACTING IN AUXETIC SILICA
}

\author{
ANDREW ALDERSON ${ }^{1}$, KIML. ALDERSON ${ }^{1}$, KENNETH E. EVANS ${ }^{2}$, \\ JOSEPH N. GRIMA ${ }^{3}$, MARK R. WILLIAMS ${ }^{1}$, AND PHILIP J. DAVIES ${ }^{1}$ \\ ${ }^{1}$ Centre for Materials Research and Innovation, Bolton Institute, Bolton BL3 SAB, UK \\ ${ }^{2}$ Department of Engineering, University of Exeter, Exeter EX4 $4 \mathrm{QF}, \mathrm{UK}$ \\ ${ }^{3}$ Department of Chemistry, University of Malta, Msida MSD 06, Malta
}

(Ree. 3 December 2004)

\begin{abstract}
Molecular mechanics simulations have been performed to undertake a systematic investigation into the structure and mechanical properties of $\alpha$-cristobalite undergoing uniaxial loading along each of the 3 mutually orthogonal principal directions and also hydrostatic pressure loading. Simulations were performed using both the BKS and Burchart force-fields. The simulations indicate that pressure loading and uniaxial loading along the $x_{3}$ direction leads to uniform variation of the four independent $\mathrm{Si}-\mathrm{O}-\mathrm{Si}$ intertetrahedral angles, indicative of cooperative tetrahedral rotation about tetrahedral axes which transform the $\alpha$-cristobalite structure into the 'idealised' $\beta$-cristobalite structure. Uniaxial loading along either of the transverse directions $\left(x_{1}\right.$ and $x_{2}$ ) leads to a divergence of the intertetrahedral angles, consistent with tetrahedral rotation about the tetrahedral axes which transform the idealised $\beta$-cristobalite structure into the 'ordered' $\beta$-cristobalite structure. The data also indicate that a phase transition to one of the proposed $\beta$ phases may be induced by a negative hydrostatic pressure or tensile stress along $x_{3}$. The phase transition is accompanied by a change in sign of some of the Poisson's ratios (i.e. from positive to negative). A negative hydrostatic pressure is also predicted to lead to conversion of initially positive to negative Poisson's ratio values (within the same phase).
\end{abstract}

\section{I N T R O D U C T I O N}

The Poisson's ratio, v, of a material or structure is defined as the ratio of the contractile transverse strain to the tensile longitudinal strain when stretched in the longitudinal direction. Most materials possess a positive $v$ and so, by definition, contract in cross-section when extended lengthwise. However, classical elasticity theory allows a material to possess a negative value of $v[1]$, that is it displays the unusual and counterintuitive behaviour of expanding laterally when stretched and contracting laterally when compressed. Such materials, known as auxetic materials [2], can have enhancements in a number of other properties (e.g. fracture toughness [3], indentation resistance [4] and acoustic response [5]) due to the presence of a negative $v$. Consequently, a host of potential applications have been identified including, for example, smart rivets, doubly-curved aircraft nose cones [6], and enhanced piezocomposite devices for naval sonar [7]. A number of synthetic auxetic materials have now been produced,

"Corresponding author: e-mail: A.Alderson@bolton.ac.uk 
including foams [3], honeycombs [8], microporous polymers [9, 10] and fibre-reinforced composites [11, 12].

At the molecular scale, a number of single-crystal materials are known to exhibit auxetic behaviour. For example, the $\alpha$-cristobalite polymorph of crystalline silica is known to be auxetic [13], and $69 \%$ of the cubic elemental metals and some $f c c$ rare gas solids are auxetic when stretched along the [110] off-axis direction [14]. One of the first attempts to design materials displaying negative Poisson's ratio behaviour at the molecular level was based on an idealised 2D system of hard cyclic hexamers [15]. This was followed by the modelling of auxetic behaviour in molecular networks based on geometries known to lead to auxetic behaviour at the macroscale, such as the design of nanoscale macrocyclic hydrocarbons based on the macroscopic re-entrant honeycomb geometry known to lead to auxetic behaviour [8]. Molecular Mechanics simulations indicated auxetic behaviour in a range of idealised zeolitic cage nanostructures [16]. Combined Molecular Mechanics and Monte Carlo simulations were used in a preliminary investigation to show that the presence of a negative Poisson's ratio enabled selective loading of neopentane and benzene guest molecules in the host zeolite MFI nanostructure through the application of an external stress in one specific direction [16].

In order to develop this area further a detailed understanding of the mechanisms and geometries necessary to realise auxetic behaviour at the nanoscale is required. In this paper, results of molecular modelling simulations on the auxetic nanomaterial $\alpha$-cristobalite are reported. The data provide greater insight into the mechanisms likely to be operating for a range of different uniaxial and pressure loading conditions.

\section{NANOSTRUCTURE OF $\alpha$-CRISTOBALITE}

The basic molecular 'building block' of $\alpha$-cristobalite is the nearly regular $\mathrm{SiO}_{4}$ tetrahedron consisting of an $\mathrm{O}$ atom at each of the four corners surrounding a central Si atom.

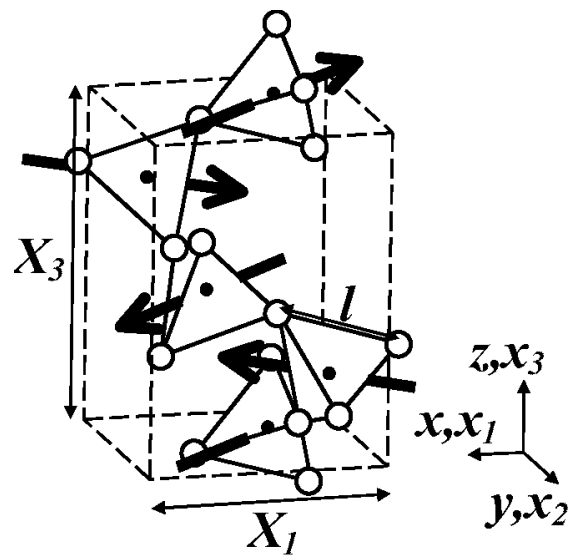

Fig. 1. Tetrahedral framework unit cell for $\alpha$-cristobalite showing tetrahedral rotation axes (solid arrows) and geometrical parameters. Filled circles are silicon atoms; empty circles are oxygen atoms 
The structure consists of a framework of corner-sharing $\mathrm{SiO}_{4}$ tetrahedra in which each $\mathrm{O}$ atom is shared between two adjacent tetrahedra. The tetragonal primitive unit-cell (space group $P 4{ }_{1} 2{ }_{1} 2$ ) contains 4 tetrahedra (Fig. 1).

\section{MOLECULAR MECHANICS MODELS}

The Cerius $^{2}$ Molecular Modelling software (Accelrys) was employed on a Silicon Graphics 02 workstation. The starting structure was as provided within the Cerius $^{2}$ structure database derived from experimental data. The modelling protocols for the structure and mechanical properties simulations were as described in detail in Ref. [16]. The stiffness matrix $\mathbf{C}$ was computed from the second derivative of the energy expression, and the on-axis Poisson's ratios and other elastic constants were obtained directly from the compliance matrix, $\mathbf{S}=\mathbf{C}^{-1}$.

Based on our previous work [16], the BKS [17] and Burchart [18] force-fields were employed in the structure and mechanical properties simulations. These force-fields were developed specifically for silicas and aluminophosphates. The BKS force-field treats interatomic interactions as ionic, with parameterisation based on both experimental and ah initio data. The Burchart force-field assumes the frameworks are largely covalent and interactions are parameterised using experimental data. Whilst these force-fields have previously [16] been found not to reproduce the negative on-axis Poisson's ratios known to occur in $\alpha$-cristobalite, they did predict low positive values and also gave reasonable agreement with the experimental on-axis Young's moduli. The BKS force-field was previously found to predict a negative average polycrystalline isotropic aggregate Poisson's ratio, as also calculated from the experimental single-crystal elastic constants.

Structure and mechanical properties simulations were performed for uniaxial loading along each of the mutually orthogonal principal axes $x_{1}, x_{2}$ and $x_{3}$ and also for hydrostatic pressure loading. Loads were applied in the range $-2 \mathrm{GPa}$ to $2 \mathrm{GPa}$ (i.e. compressive and tensile loads were considered).

\section{RESULTS}

Table 1 shows a comparison of the experimental (undeformed) and predicted unit-cell dimensions for $\alpha$-cristobalite. The Burchart force-field generally gives marginally better agreement with the experimentally-determined undeformed unit-cell lengths, and is in particularly good agreement with the values of the transverse unit cell dimensions $\left(X_{1}\right.$ and $X_{2}$, see Fig. 1). The greatest discrepancy between the predicted and experimental values occurs for the BKS predicted value of $X_{3}$, which is $\sim 6 \%$ lower than the experimental value. The predicted values are similar to those from the pair potential calculations of Keskar and Chelikowsky [19] who reported predicted values of $X_{1}=4.96 \AA$ and $X_{3}=6.68 \AA$. 
Table 1. Experimental and predicted unit-cell lengths (A) from the Molecular Mechanics simulations for $\alpha$-cristobalite. Modelling data are shown for simulations employing the Burchart and BKS force-fields with no external applied stress, and also for the BKS force-field with a negative hydrostatic pressure of $-0.844 \mathrm{GPa}$ applied

\begin{tabular}{lcccc}
\hline Unit-cell length & $\begin{array}{c}\text { Experiment } \\
{[20]}\end{array}$ & Burchart & BKS & $\begin{array}{c}\text { BKS } \\
(p=-0.844 \text { GPa })\end{array}$ \\
\hline$X_{1}=X_{2}$ & 4.978 & 4.982 & 4.889 & 4.946 \\
$X_{3}$ & 6.948 & 6.658 & 6.529 & 6.663 \\
\hline
\end{tabular}

The experimental (undeformed) mechanical properties (Poisson's ratios and Young's moduli) and the predicted mechanical properties from the Molecular Mechanics simulations are presented in Table 2. The Burchart and BKS force-field data are in close agreement with those from our earlier simulations [16], predicting low positive values (but not negative) for the on-axis Poisson's ratios measured experimentally to be negative in the $x_{1}-x_{3}$ plane $\left(\mathrm{v}_{13}\right.$ and $\mathrm{v}_{31}$ ). The Burchart force-field tends to be the better force-field for the prediction of the Poisson's ratios, whereas the BKS force-field gives closer agreement in the Young's moduli.

Table 2. Molecular Model and experimental mechanical properties for $\alpha$-cristobalite. Numbers in parentheses are uncertainty in least significant figure

\begin{tabular}{lcccc}
\hline & $\begin{array}{c}\text { Experiment } \\
{[13]}\end{array}$ & Burchart & BKS & $\begin{array}{c}\text { BKS } \\
(p=-0.844 \mathrm{GPa})\end{array}$ \\
\hline$v_{12}$ & $+0.06(1)$ & +0.19 & +0.16 & +0.11 \\
$v_{13}$ & $-0.10(2)$ & +0.04 & +0.08 & -0.10 \\
$v_{31}$ & $-0.07(1)$ & +0.02 & +0.05 & -0.06 \\
$E_{1}[\mathrm{GPa}]$ & $58.8(5)$ & 104.6 & 67.8 & 59.0 \\
$E_{3}[\mathrm{GPa}]$ & $41.8(7)$ & 44.8 & 46.8 & 37.4 \\
\hline
\end{tabular}

Figure 2 shows the predicted variation of the four independent $\mathrm{Si}-\mathrm{O}$-Si angles in $\alpha$-cristobalite in response to uniaxial loading in each of the 3 principal directions and also due to hydrostatic pressure loading for the BKS force-field simulations. Similar trends were observed in the Burchart force-field simulations. In the unloaded structure the four angles are equal to each other. In the case of hydrostatic pressure loading (Fig. 2a) and also uniaxial loading along the $x_{3}$ direction (Fig. 2b) the four angles remain equal to each other but vary with applied load. However, for uniaxial loading along the $x_{1}$ and $x_{2}$ direction there is a divergence between one pair of angles and another (Figs. $2 \mathrm{c}$ and $2 \mathrm{~d}$ ). The simulations also indicated that the tetrahedra undergo a change in size when a load is applied, which is also therefore a deformation mechanism in these cases.

Figure 2a also indicates that a phase transition is predicted for a negative (i.e. tensile) applied hydrostatic pressure of $-1.7 \mathrm{GPa}$. From observation of the structural data, the 
transition is predicted to be from $\alpha$-cristobalite to the 'idealised' $\beta$-cristobalite structure proposed by Wyckoff [21].
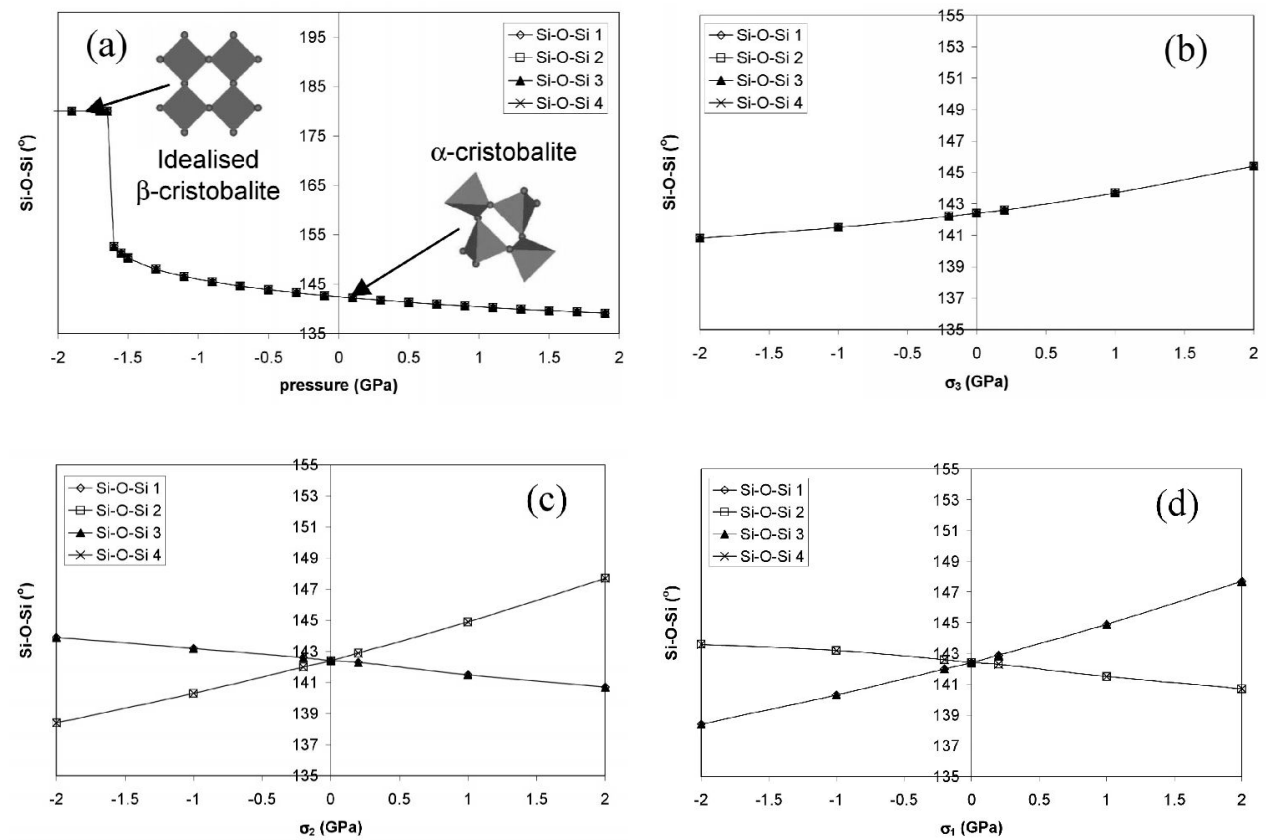

Fig. 2. Si-O-Si intertetrahedral bond angle versus applied load from the BKS force-field simulations of $\alpha$-cristobalite: (a) hydrostatic pressure - inserts show $x_{1}-x_{2}$ plane projections of unit-cell corresponding to $\alpha$-cristobalite and 'idealised' $\beta$-cristobalite; (b) stress along the $x_{3}$ direction; (c) stress along the $x_{2}$ direction; (d) stress along the $x_{1}$ direction
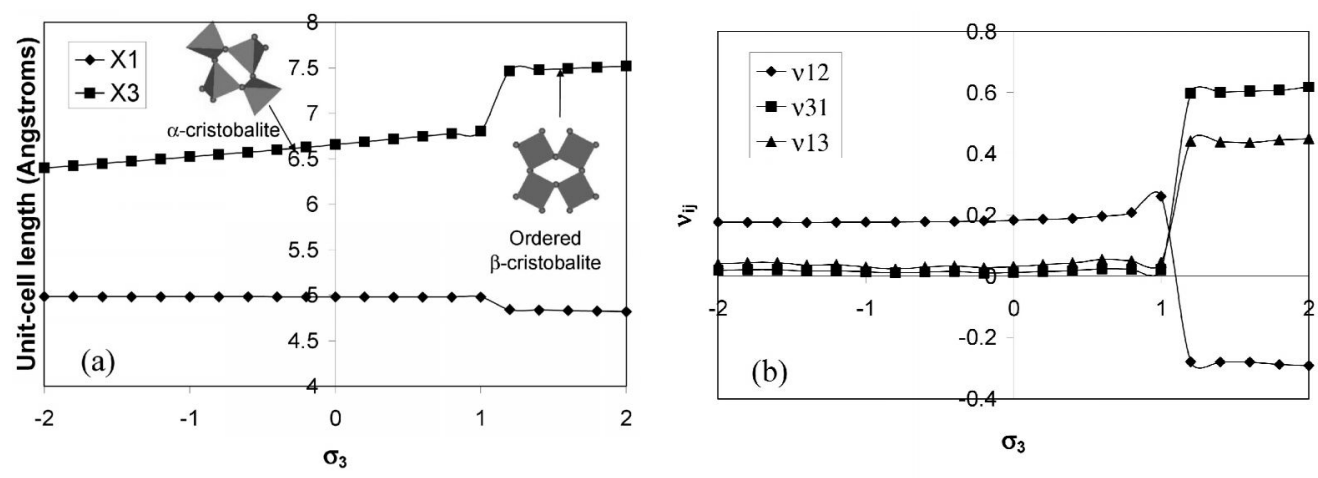

Fig. 3. Burchart force-field simulation data for $\alpha$-cristobalite: (a) unit-cell lengths $X_{1}$, and $X_{3}$ versus $\sigma_{3}$ - inserts show $x_{1}-x_{2}$ plane projections of unit-cell corresponding to $\alpha$-cristobalite and 'ordered' $\beta$-cristobalite; (b) $\mathrm{v}_{i j}$ versus $\sigma_{3}$ 
Figure 3a shows the calculated unit-cell parameter variations with loading along $x_{3}$ predicted from the Burchart force-field simulations. The unit-cell length $X_{I}$ remains nearly constant with applied load, consistent with the low positive (near zero) value of $v_{31}$ calculated using the Burchart force-field (Table 2). A phase transition is predicted at a stress value within the range of $1 \mathrm{GPa}<\sigma_{3}<1.2 \mathrm{GPa}$. The inserts in Fig. 3a show the $x_{1}-x_{2}$ projections for the unit-cell and correspond to $\alpha$-cristobalite and 'ordered' $\beta$-cristobalite (proposed by Wright and Leadbetter [22]) for the compressive/low tensile and high tensile stress regions, respectively.

Figure $3 \mathrm{~b}$ shows the predicted $\sigma_{3}$-dependent Poisson's ratio behaviour from the Burchart force-field simulations. The Poisson's ratios show a clear change in magnitude from one phase to the other, and include a change in sign of $v_{12}$ from positive ( $\alpha$-cristobalite) to negative ('ordered' $\beta$-cristobalite).

Figures $4 \mathrm{a}$ and $4 \mathrm{~b}$ show the predicted effect of pressure on the Poisson's ratios and Young's moduli, respectively, for $\alpha$-cristobalite using the BKS force-field. The experimental data are also indicated at $p=0$ for comparison. The BKS force-field consistently overestimates the experimental elastic constants at zero pressure and predicts all on-axis Poisson's ratios to be positive (see also Table 2). A negative hydrostatic pressure (i.e. isotropic tension) leads to a decrease in the magnitude of the predicted elastic constants, and leads to conversion of the initially positive Poisson's ratios to negative values.
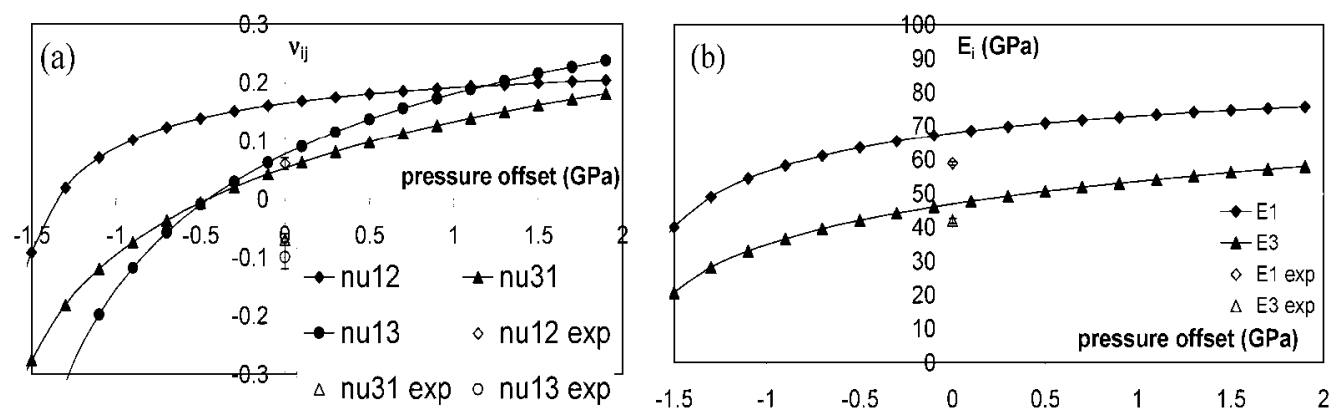

Fig. 4. (a) $v_{i j}$ and (b) $E_{i}$ variations $v$ hydrostatic pressure offset (BKS force-field) for $\alpha$-cristobalite. Experimental data for undeformed $\alpha$-cristobalite are also shown (empty symbols)

An improved Molecular Mechanics model for the 'undeformed' structure, comprising the BKS force-field with a negative hydrostatic pressure offset of $-0.844 \mathrm{GPa}$, has thus been devised. With this offset, the agreement between the Molecular Mechanics model and experimental unit-cell dimensions and elastic constants is excellent (Tables 1 and 2). Whilst improving the agreement in the absolute values of the structural and mechanical properties, the 
Fig. 5: Si-O-Si intertetrahedral bond angle versus applied stress along the $\mathrm{x}_{\mathrm{t}}$ direction from the BKS force-field simulations of a-cristobalite with a hydrostatic pressure offset of $-0.844 \mathrm{GPa}$ applied

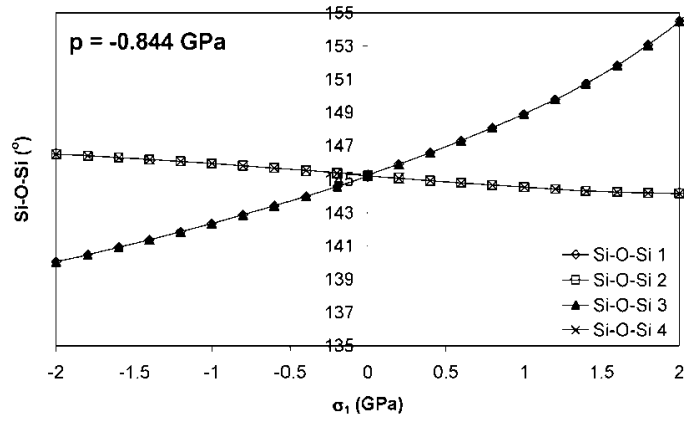

presence of a hydrostatic pressure offset of $-0.844 \mathrm{GPa}$ does not alter the predicted trends in the bond lengths and angles with applied load, compare for example the Si-O-Si variation with $\sigma_{1}$ with and without a pressure offset of $-0.844 \mathrm{GPa}$ (Figs. 5 and $2 \mathrm{~d}$, respectively).

\section{DISCUSSION}

The above comparison of the unit-cell lengths and mechanical properties of undeformed $\alpha$-cristobalite predicted in this project with previous experimental and theoretical data confirms that the modelling protocol employed is appropriate.

The intertetrahedral angle is a function of the orientation of the tetrahedra (tilt angle) about the axes shown in Fig. 1 [23]. Hence the uniform variation of all 4 independent Si-O-Si angles shown in Figs. $2 a$ and $2 b$ indicate that cooperative tetrahedral rotation about the axes shown in Fig. 1 is a deformation mechanism for $\alpha$-cristobalite when subject to hydrostatic pressure or $\sigma_{3}$ loading. This is consistent with our previously developed analytical models which have demonstrated that dilation of the tetrahedra acting concurrently with cooperative rotation of the tetrahedra about the axes shown in Fig. 1, and the equivalent axes in the $\alpha$-quartz nanostructure, accurately predicts the negative and positive values of $\mathrm{v}_{31}$ for a-cristobalite and $\alpha$-quartz, respectively [24].

Divergence of the Si-O-Si angles shown in Figures $2 \mathrm{c}$ and $2 \mathrm{~d}$ indicates that an alternative deformation mechanism operates for uniaxial loading of $\alpha$-cristobalite in a transverse direction $\left(x_{1}\right.$ and $\left.x_{2}\right)$, explaining why the above analytical model fails to accurately predict the values of

$\mathrm{v}_{12}$ and $\mathrm{v}_{13}$ [24]. Based on these molecular modelling results we have recently extended the analytical models to incorporate a second tetrahedral rotation system [25]. Figure 6 shows both tetrahedral rotation systems considered as a result of the molecular model data. Figure 6a is the projection onto the $x_{1}-x_{2}$ plane of the unit-cell of $\alpha$-cristobalite with the tilt axes of Fig. 1 indicated. The projection of the 'untilted' (tilt angle $S=0$ ) structure in the $x_{1}-x_{2}$ plane is shown in Fig. $6 \mathrm{~b}$ and corresponds to the 'idealised' $\beta$-Cristobalite structure (space group Fd3m) originally proposed by Wyckoff [21] for the high temperature phase of cristobalite. Rotation of each tetrahedron in the idealized $\beta$-cristobalite structure by an angle $\quad\left(=19.8^{\circ}\right)$ 
about a tetrahedral axis aligned along the $\mathrm{x}_{3}$ direction (i.e. perpendicular to the $x_{1}-x_{2}$ plane) transforms the structure into that of 'ordered' $\beta$-cristobalite [22] (space group $142 d$ ) - Fig. 6c. It is this second tetrahedral rotation mechanism (Fig. 6c) superimposed onto the $\alpha$-cristobalite structure (Fig. 6a) which is indicated by the Molecular Mechanics simulations as operating when loading $\alpha$-cristobalite in the transverse direction $\left(x_{1}\right.$ or $\left.x_{2}\right)$. The analytical model in which both tetrahedral rotation systems act concurrently with tetrahedral dilation has been found to accurately reproduce the values of $v_{12}$ and $v_{13}$ due to uniaxial loading of $\alpha$-cristobalite along $x_{1}[25]$.

It is interesting that the BKS force-field predicts an a-cristobalite to 'idealised' $\beta$-cristobalite phase transition for negative (tensile) hydrostatic pressure loading (Fig. 2a) whereas the Burchart force-field predicts an a-cristobalite to 'ordered' $\beta$-Cristobalite phase transition for uniaxial tensile loading along $\mathrm{x}_{3}$. The actual structure of $\beta$-Cristobalite remains a topic of some debate in the literature [22, 23, 26-28]. The analytical model of the first tetrahedral rotation mechanism (Fig. 6a) acting concurrently with tetrahedral rotation has been shown to predict a $\sigma_{3}$-induced $2^{\text {nd }}$ phase consistent with the 'idealised' $\beta$-Cristobalite structure $[29,30]$. However, since this particular analytical model does not include the second rotation system it cannot predict the 'ordered' $\beta$-Cristobalite structure.

The predicted change in sign of $v_{12}$ at the phase transition from positive ( $\alpha$-cristobalite) to negative ('ordered' $\beta$-cristobalite) in Fig. $3 b$ has been observed in other materials. For example, the Poisson's ratio of poly(N-isopropylacrylamide)/water gel has been observed to change from a negative to a positive value at the volume phase transition [31].

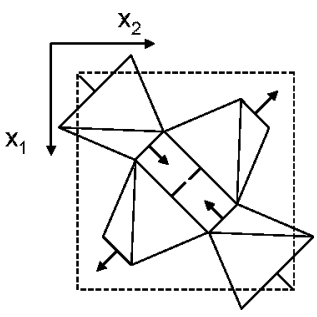

$\alpha$-cristobalite

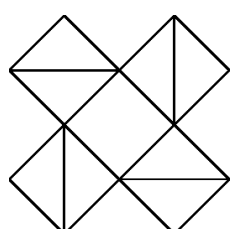

'Idealised' $\beta$-cristobalite

(b)

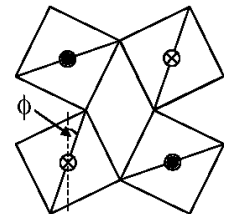

'Ordered'

$\beta$-cristobalite

(c)

Fig. 6. $x_{1}-x_{2}$ projections of: a) $\alpha$-cristobalite, b) idealised $\beta$-cristobalite and c) ordered $\beta$-cristobalite

Figure 4 demonstrates the predicted positive Poisson's ratio in the undeformed structure is transformed into a negative Poisson's ratio at a sufficiently negative (tensile) value of applied pressure. The role of a negative pressure in producing negative Poisson's ratios in molecular systems has previously been reported for the case of an $f \mathrm{cc}$ tethered crystal [32]. 


\section{CONCLUSIONS}

The systematic study, using molecular mechanics simulations, of the deformation of $\alpha$-cristobalite under a variety of loading conditions suggests that $\alpha$-cristobalite deforms by:

- 1 cooperative tetrahedral rotation system (about the tetrahedral axes shown in Fig. 6a) acting concurrently with tetrahedral dilation for uniaxial loading along the $x_{3}$ direction and also hydrostatic pressure laoding.

2 cooperative tetrahedral rotation systems (about the axes shown in Figs. 6a and 6c) acting concurrently with tetrahedral dilation for uniaxial loading along the $x_{1}$ (and $x_{2}$ ) direction.

Negative hydrostatic pressure loading is predicted to transform initially positive to negative Poisson's ratios and eventually leads to a phase transition (BKS force-field). The Burchart force-field simulations indicate a phase transition may be induced by tensile loading along $x_{3}$.

\section{Acknowledgements}

The authors would like to thank EPSRC for funding this work.

\section{References}

[1] A. E. H. Love, A Treatise on the Mathematical Theorv of Elasticity, 4th ed.; Dover: New York (1944)

[2] K. E. Evans, M. M. Nkansah, I. J. Hutchinson, and S. C. Rogers, Nature 353, 124 (1991).

[3] R. S. Lakes, Science 235, 1038 (1987).

[4] K. L. Alderson, A. P. Pickles, P. J. Neale, and K. E. Evans, Acta Metall. Mater. 42, 2261 (1994).

[5] A. W. Lipsett and A. I. Beltzer, J. Acoust. Soc. Am. 84, 2179 (1988).

[6] K. E. Evans, Tailoring the Negative Poisson's Ratio. Chem. Ind. 20, 654 (1990).

[7] W. A. Smith, in:' Proceedings of IEEE ultrasonics symposium IEEE, 661 (1991).

[8] L. J. Gibson, and M. F. Ashbv, in: Cellular Solids: Structure and Properties (Pergamon Press, Oxford, 1988).

[9] B. D. Caddock and K. E. Evans, J. Phvs. D: Appl. Phvs. 22, 1877 (1989).

[10] K. L. Alderson, and K. E. Evans, Polymer 33, 4435 (1992).

[11] J. P. Donoghue and K. E. Evans, in: Proc. ICCM 8 (Eds. S. W. Tsai, G. S. Springer), S WIPE. Covina, CA, (1991), 2-K-1.

[12] J. F. Clarke, R. A. Duckett, P. J. Hine, I. J. Hutchinson, and I. M. Ward, Composites 25, 863 (1994).

[13] Y. Yeganeh-Haeri, D. J. Weidner, and J. B. Parise, Science 257, 650 (1992).

[14] R. H. Baughman, J. M. Shacklette, A. A. Zakhidov, and S. Stafstrom, Nature 392, 362 (1998).

[15] K. W. Wojciechowski, Molecular Physics 61, 1247 (1987).

[16] J. N. Grima, R. Jackson, A. Alderson, and K. E. Evans: Adv. Mater. 12 (24), 1912 (2000).

[17] B. W. H. Van Beest, G. J. Kramer, and R. A. Van Santen: Phys. Rev. Lett. 64, 1955 (1990).

[18] E. Vos Burchart, in: Studies on Zeolites: Molecular Mechanics, Framework Stability and Crystal Growth, Ph.D. Thesis, Delft University of Technology, The Netherlands 1992, Table I, Chapter XII.

[19] N. R. Keskar and J. R. Chelikowsky, Phys. Rev. B, 46, 1-13 (1992).

[20] W. A. Dollase, Z. Kristallogr. 121, 369 (1965).

[21] R. W. G. Wyckoff, Amer. J. Sci. 9, 448-459 (1925).

[22] A. F. Wright and A. J. Leadbetter, Phil. Mag. 31, 1391-1401 (1975).

[23] M. O'Keeffe and B. G. Hvde: Acta Crvst. B 32, 2923 (1976). 
[24] A. Alderson and K. E. Evans, Phys. Rev. Lett. 89(22) 225503-1 (2002).

[25] A. Alderson, K. L. Alderson, K. E. Evans, J. N. Grima, M. R. Williams, and P. J. Davies. Physica Status Solidi, (2004), submitted.

[26] A. J. Leadbetter, T. W. Smith and A. F. Wright, Nature 244, 125-126 (1973).

[27] W. Nieuwenkamp, Z. Kristallogr. 96, 454 (1937).

[28] D. M. Hatch and S. Ghose, Phys. Chem. Minerals, 17, 554-562 (1991).

[29] A. Alderson and K. E. Evans, Phys. Rev. B. Submitted (2004).

[30] A. Alderson, K. L. Alderson, K. E. Evans, J. N. Grima, and M. Williams, Journal of Metastable and Nanocrvstalline Materials, 23, 55-58 (2004).

[31] S. Hirotsu, J Chem Phys. 94, 3949 (1991).

[32] K. W. Wojciechowski and K. V. Tretiakov, Comp. Meth. Sei. Tech. 1, 25 (1996). 